\title{
Imaging the Brain In Situ with Phase Contrast CT
}

Linda Croton ${ }^{1, *}$, Kaye Morgan ${ }^{1,2}$, David Paganin ${ }^{1}$, Lauren Kerr, ${ }^{3,4}$, Megan Wallace ${ }^{3,5}$, Kelly Crossley ${ }^{3,5}$, Gary Ruben $^{1}$, Suzanne Miller ${ }^{3}$, Naoto Yagi ${ }^{6}$, Kentaro Uesugi $^{6}$, Stuart Hooper ${ }^{3,5}$, Marcus Kitchen ${ }^{1}$

1. School of Physics and Astronomy, Monash University, Clayton, Victoria 3800, Australia

2. Chair of Biomedical Physics, Department of Physics, Munich School of Bioengineering, and Institute of Advanced Study, Technische Universität München, 85748 Garching, Germany

3. The Ritchie Centre, Hudson Institute of Medical Research, Clayton, Victoria 3800, Australia

4. Cancer Research UK, Angel, London, United Kingdom

${ }^{5 .}$ Department of Obstetrics and Gynaecology, Monash University, Clayton, Victoria 3800, Australia

6. Japan Synchrotron Radiation Research Institute (JASRI/SPring-8), 1-1-1 Kouto, Sayo, Hyogo 6795198, Japan

*linda.croton@monash.edu

Phase contrast X-ray imaging (PCXI) is an emerging modality that exploits the differing refractive indices of materials to create additional image contrast. When used in conjunction with computed tomography (CT), PCXI can produce images with greatly increased contrast resolution with respect to standard attenuation CT. This means that soft tissue boundaries that are typically not well resolved, such as those between the grey and white matter in the brain, can be visualized clearly. The brain poses unique problems for PCXI-CT, since it is fully encased in the highly-attenuating skull. Damage or defects in the imaging system and other physical effects result in inaccurate estimates of the attenuation gradient across high-contrast boundaries, causing distinct streak artifacts that can overwhelm the parts of the image that contain underlying tissues. In addition, ring artifacts caused by variations in X-ray beam intensity and detector response are more difficult to remove due to the roughly circular symmetry of the skull. Many common ring removal methods exploit the circular symmetry seen in ring artifacts (see [1] and [2] for examples) and cannot distinguish between the skull and these artifacts.

The causes of streak artifacts are many and varied and include the detector point spread function, edge gradient effects, Compton scattering, beam hardening, photon starvation, Poisson noise, and the higherorder harmonics of synchrotron monochromator crystals [3]. Taking all of these effects into account simultaneously is a very difficult process and hinders the development of a single, general algorithm to correct for them. We show that these artifacts can be avoided with relative ease and that the same circular symmetry that complicates ring artifact correction also reduces the effect that streak artifacts have in obscuring the brain. We present a simple and effective ring artifact correction that is easy to implement and does not require a priori knowledge of the sample and show that it suppresses artifacts to a level that makes a substantial difference for phase contrast CT.

CTs were acquired of a New Zealand White rabbit kitten head suspended in agarose at both an $11 \mathrm{~cm}$ and a $5 \mathrm{~m}$ sample-to-detector propagation distance on beamline 20BL2 at the SPring-8 synchrotron in Japan. The sample orientation during CT acquisition placed the sagittal and coronal axes nearly vertical. Each detector row was reconstructed independently and the full volume reoriented to create new volumes parallel to the axial, sagittal, and coronal axes. Figure 1 shows a comparison between an $11 \mathrm{~cm}$ propagated view (effectively attenuation $\mathrm{CT}$ ) and a $5 \mathrm{~m}$ propagated view after phase retrieval for a sagittal slice near the midline of the brain ( $\mathrm{px}=25 \mu \mathrm{m}, 3600$ projections). Phase retrieval was performed using a two-material algorithm, derived from that given in [4], now for the specific case of CT, such that 
knowledge of the full sample thickness is no longer required. A clear difference can be seen between the attenuation contrast image, where no discernible features are visible, and the phase image, where the grey/white boundaries are clearly delineated. In particular, the cerebellum $(\mathrm{Cb})$ is quite well resolved on the right side of the phase-retrieved image. By orienting the sample at a slight angle with respect to the standard imaging planes, streak artifacts are minimized in those planes, since they are most visible in the offset plane of reconstruction. The ring artifacts are also minimized; however, they can still be seen as a diffuse band extending diagonally upward, in the direction of the arrow, toward the cerebellum.

To minimize these ring artifacts, we create a pixel-wise detector calibration to estimate the true intensity incident on the detector in lieu of a standard dark current and flat-field correction, with an adjustment for temporal fluctuations in the beam intensity and for experiment-specific deviations from the calibration. Figure 2 shows a phase-retrieved slice ( $\mathrm{px}=15 \mu \mathrm{m}, 1800$ projections), reconstructed after standard dark current and flat-field correction (left) and the same slice with our method implemented (right).

These results demonstrate the first in situ brain imaging of small animals using PCXI-CT, showing a significant increase in signal-to-noise and revealing subtle brain features deep within the skull. Using a two-material phase retrieval algorithm, we obtain images with greatly improved image contrast compared to the single-material algorithm frequently used [5]. Applying a simple artifact correction enhances these features, further improving the definition to reveal additional detail.

\section{References:}

[1] B Münch et al, Opt. Express 17 (2009) 8567

[2] D Prell, Y Kyriakou and WA Kalender, Phys. Med. Biol. 54 (2009) 3881

[3] L Croton et al, arXiv:1801.07968, submitted (2018)

[4] M Beltran et al, Opt. Express 18 (2010) 6423

[5] D Paganin et al, Opt. Comm. 234 (2002) 87

[6] This work was funded by ARC Discovery Project DP170103678 and supported by SPring-8 proposals 2015B0047 and 2016A0047. The authors acknowledge travel funding from the Australian Synchrotron Access Program (ISAP) managed by the Australian Synchrotron and funded by the Australian Government (AS/IA153/10571).

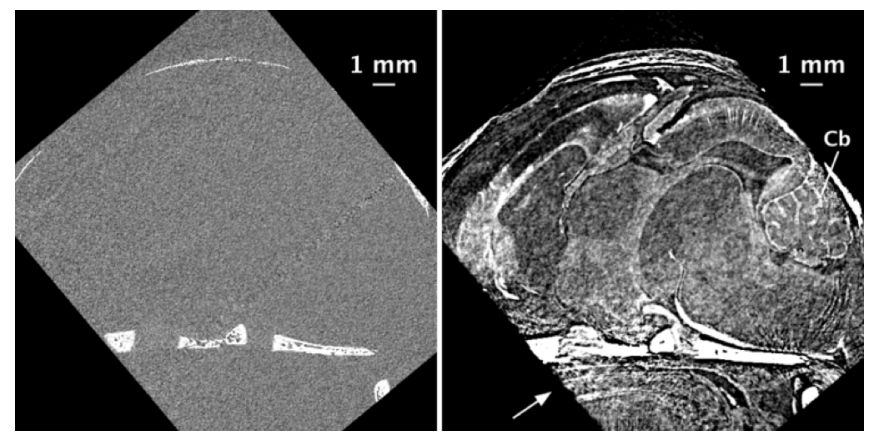

Figure. 1. Sagittal view of a rabbit kitten brain in situ at an $11 \mathrm{~cm}$ (left) and $5 \mathrm{~m}$ (right, after phase retrieval) sample-to-detector propagation distances at an energy of $24 \mathrm{keV}$. The arrow denotes the position and direction of the ring artifacts across the sagittal plane toward the cerebellum $(\mathrm{Cb})$.

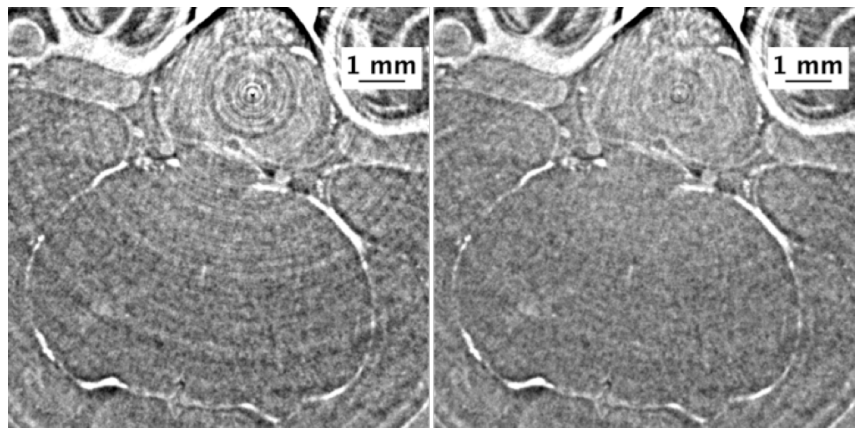

Figure. 2. Phase-retrieved, propagation-based tomograms of a rabbit kitten brain in situ, taken at a $5 \mathrm{~m}$ sample-to-detector propagation distance at $24 \mathrm{keV}$. The left image shows the ring artifacts before correction, and the right image has been corrected using our pixel-wise detector calibration. 